\title{
Oxytocin and emotion processing
}

Di Simplicio M. ${ }^{1}$ and Harmer C.J. ${ }^{2}$

${ }^{1}$ MRC Cognition and Brain Sciences Unit, Cambridge, UK

${ }^{2}$ Department of Psychiatry, University of Oxford, Oxford, UK

\section{ABSTRACT}

Since the observation that oxytocin has key effects on social decision making, research on this exciting neuropeptide has doubled in volume: hundreds of studies have pursued the promise of a specific oxytocin action on high-level cognition and social function with wide potential translational implications (from autism to social anxiety to dementia). Here we review the evidence on whether the complex behavioural effects observed in humans after exogenous oxytocin administration build on changes in basic emotional information processing, in particular emotional facial expressions recognition, and attention and memory for emotionally-valenced stimuli.

We observe that recent studies confirm a facilitatory effect of oxytocin to more accurate emotion processing, irrespective of emotion type. However, it remains unclear whether this action precedes, is independent of or even secondary to the neuropeptide promoting a greater salience of social stimuli. Overall, this growing research area has shown that oxytocin produces behavioural and neurofunctional outcomes that are highly dependent on the experimental context and on individual differences (gender, personality, life experiences). This poses an exciting challenge for future experimental medicine designs to address and unpack complex interactions between individual and context characteristic, which is needed for the development of more precise clinical applications. 
The study of the neuropeptide oxytocin and its effects on animal and human social behaviour has become one of the most popular areas of psychopharmacology research over the last ten years. The number of publications in the field has boomed, for example going from 350 hits on PubMed for 'oxytocin and human behaviour' in 2005-2010 to 745 hits in 2010-2015. This is unsurprising given the exciting promise that oxytocin may exert a specific action on high-level cognition and social function. The potential translational implications, recently extended from autism and social anxiety to PTSD and borderline personality disorder or dementia, further justify the success and popularity of this relatively new field of research.

Our 2009 paper Oxytocin enhances processing of positive vs negative emotional information in healthy male volunteers described the effect of a single dose intranasal administration of oxytocin on the processing of emotional information in a sample of healthy volunteers. Our curiosity - as that of many others - was triggered by the seminal experiment of Kosfeld et al. (2005) that revealed increased trust-related behavior after just a single dose of intranasal oxytocin administration compared to placebo.

Our study assessed whether oxytocin's effects on social behaviour could be underpinned by changes in emotional information processing, similar to the growing evidence that antidepressant effects on mood and behaviour may derive from reverting negative cognitiveemotional biases typical of depression (Warren et al 2015). In particular, we used the same experimental methodology previously used to elucidate antidepressant drug action to explore the effects of oxytocin (Harmer et al., 2003, 2004, Browning et al., 2007). Comparing a single dose of intranasal oxytocin (24UI) to placebo in healthy male volunteers, we showed that oxytocin modulated the recognition of emotional facial expressions. The effects were subtler than seen with antidepressant drug treatment, but broadly in the same direction. That is, that oxytocin facilitated the accurate identification of positive vs. negative emotions and slowed down the recognition of negative emotional expressions such as morphed fearful faces.

Further studies have addressed the same question following our findings in larger samples and including female participants as well as males. Using a very similar emotion recognition paradigm, Marsh et al. (2010) found that oxytocin produced a more accurate recognition of morphed happy faces compared to placebo. However, some studies also reported an oxytocin-induced in negative bias in emotional facial expression processing (Fischer-Shofty et al., 2010, Lischke et al., 2012a). A recent meta-analysis by Shahrestani et al. (2013) confirmed that single dose intranasal oxytocin administration to healthy volunteers facilitates emotion recognition relative to placebo, regardless of facial expression type. This effect appeared to be of small size and moderated by emotion type, so that oxytocin had a significant impact on happy faces under implicit recognition parameters $(<300 \mathrm{~ms}$ presentation duration) and on fearful faces under explicit recognition parameters ( $>300 \mathrm{~ms})$. The latter is consistent with data on happy faces being easier to recognise, so that with longer presentation duration accuracy scores may reach a ceiling effect. It should be noted that while a quantitative meta-analysis represents a progress in our understanding of the current literature, this was only able to include seven out of the numerous papers that have investigated facial expression processing as it was limited to data reporting accuracy outcomes while processing full faces of $100 \%$ emotional intensity. Further experimental 
studies are therefore required to draw definitive conclusions on the presence/absence of a valence-based modulation of facial expressions processing by oxytocin.

The lack of a valence-based effect on facial expression processing following oxytocin administration may appear paradoxical: the numerous accounts of oxytocin facilitating prosocial and approach behaviour may be predicted to rely on increased positive bias processing (i.e. a selective focus on approach relevant emotions such as happiness). However, a prosocial outcome may also result from increasing the tendency to correctly process all emotional expressions and in the reduction of ambiguity, which in turn may promote feeling at ease with facial expressions regardless of their emotion, increase empathy (Hurlemann et al., 2010, Bartz et al., 2010) and even reduce the natural aversion to negative faces (Evans et al., 2010). Therefore, consistent with our original hypothesis, it is possible that increased processing of emotional stimuli, in particular recognition of facial expressions, contributes to changes in more complex social behaviour. This link needs to be tested for example, by using mediation analysis in large studies capturing both changes with oxytocin administration.

In our original study, we also tested whether oxytocin administration produced any emotional attentional bias compared to placebo, using a dot probe task with happy and fearful faces. While the same task has been shown to be sensitive to antidepressant and benzodiazepines manipulation (Browning et al., 2007, Murphy et al., 2008), oxytocin did not appear to modify covert attention to emotional stimuli in this paradigm. Based on null results on a visual search task, Guastella and MacLeod (2012) also concluded that oxytocin was unlikely to act via shifting attention towards or away from social/emotional stimuli. However, subsequent studies have shown that oxytocin is able to modulate early attentional processing towards emotional stimuli (Domes et al., 2013, Clark-Elford et al., 2014, Tollenaar et al., 2013). Increased eye gaze exploration of the eye region of faces following oxytocin administration has also been reported, although results are inconsistent and possibly task, population or gender-dependent (Gamer et al., 2010, Andari et al., 2010, Guastella et al., 2008, Domes et al., 2009).

Attention bias is one of the mechanisms believed to underpin symptoms of anxiety, and the findings above suggest that oxytocin may have an anxiolytic effect by shifting attention towards positive cues. Moreover, if anxiety is characterised by misinterpretation of stimuli as threatening, oxytocin may reduce this by promoting the exploration of any salient facial expression and possibly increasing engagement with social stimuli (in opposition to avoidance mechanisms that maintain anxiety). Therefore anxiety reduction - at least social anxiety - may result from both valence and non-valence-based changes in attention mechanisms. It has been suggested that the so-called oxytocin 'prosocial' effects on higher order cognitions and behaviour may be secondary to such general anxiety reduction. This hypothesis is further backed by evidence of oxytocin also having an impact on arousal (Ellebogen et al., 2014) and other measures of stress reactivity unrelated to social stimuli (for a review see Neumann and Slattery, 2016); however, there are also accounts of oxytocin-induced increases in anxiety (Grillon et al., 2013).

An alternative view around oxytocin's mechanism of action argues that this occurs by increasing the salience and processing specifically of social stimuli (positive and negative), possibly via interaction with the dopaminergic mesocortico-limbic system (for a review see Shamay-Tsoory and Abu-Akel, 2015) and unrelated to any anxiolytic effect. This social 
salience hypothesis could equally explain the above findings on facial expression recognition and attentional biases: increased attention to facial features that convey a social meaning (eyes, mouth) following oxytocin administration (Guastella et al., 2008, Gamer et al., 2010) could in turn facilitate emotion recognition regardless of emotion type. One study that addressed this idea found that improved emotion recognition was not associated with changes in visual scanning of faces (Lischke et al., 2012a). Neuroimaging data have indicated that compared to placebo oxytocin was associated with differential reactivity to emotional facial expression and to eye gaze deployment to facial features (irrespective of emotion) in different subregions of the amygdala (Gamer et al., 2010). Another study failed to detect eye gaze shift in correspondence with increased amygdala reactivity to threatening scenes (Lischke et al., 2012b), but this could also suggest that modulation of eye gaze orientation is specific to face processing, faces and eyes being the 'most' social of human stimuli.

Overall, the above evidence suggests that the oxytocin-driven modulation of emotion processing may actually be separate if not secondary to the facilitation of social cues processing. A difficulty in disentangling these effects derives from the obvious fact that social stimuli very often convey an emotional content. Most emotional cues used in human experimental designs are of a social nature and most paradigms to date have utilised mostly both positive and negative (rather than neutral) social stimuli compared to non-social stimuli to demonstrate the specificity of oxytocin's action on social salience (but see Perry et al., 2010 for a task where oxytocin modulated the response to biological compared to nonbiological motion). Advocates of the social salience hypothesis highlight that this allows us to reconcile accounts of oxytocin promoting 'antisocial' effects, because increased salience attribution to social cues may well result in expressing emotions of envy (Shamay-Tsoory et al., 2009), hindering trust (Bartz et al., 2011a) or inhibiting disengagement from sad faces (Ellenbogen et al., 2013). Overall, while the view that oxytocin exclusively promotes prosocial affiliative behaviour via producing a positive emotional bias - in particular in facial expression processing - appears simplistic, the association and hierarchy of oxytocin effects on emotional and social stimuli remains to be fully understood.

Finally, our study did not detect changes in memory for self-relevant emotional stimuli by oxytocin compared to placebo. Subsequent research on animal models and human participants has shown that by using fear and social fear conditioning paradigms oxytocin's effects on memory can be dissected into a complex picture whereby results are dependent on the phase of peptide administration (pre vs post memory encoding) and the nature of the conditioning stimulus. Animal studies suggest that oxytocin influence on memory acquisition and extinction may also be dose-dependent, although this remains to be tested in humans (see for a review Chini et al., 2014). While effects on memory and fear do not appear to be specific for social stimuli, it has been hypothesised that oxytocin role in social recognition memory and fear responses is also mediated by changing the emotional and social saliency of stimuli and contexts (Maroun and Wagner, 2016).

In summary, the explosion of research on oxytocin's effects on emotion processing and investigation of the other potential mechanisms that could underpin the peptide's role in human behaviour depicts a complex, and at times, contradictory landscape. Leaving aside the debate on if and how exogenous intranasal oxytocin reaches brain sites of action (Leng and Ludwig, 2016), some inconsistencies are probably the result of methodological shortcomings and small sample sizes (Walum et al., 2016, Guastella and MacLeod, 2012). 
Unlike data on antidepressant administration, there isn't yet a robust set of tasks that could assess response to oxytocin administration in a replicable way, and this should be one of the aims for future research, as already highlighted by others (Sharestani et al., 2013). Another issue is some confusion in the level of investigation and explanation that is sometimes adopted. For example, it is frequently reported that experiments 'controlled for anxiety' and oxytocin did not exert any subjective change in anxiety, however this does not exclude that the peptide may still have acted via modifying preconscious cognitive processes that contribute to subjective anxiety, and so via reducing the likelihood of anxiety formation. A major challenge remains to determine the links between the different stages of cognitive processing and their underpinning neural circuitry (Guastella and Macleod, 2012), and whether the modulation of more basic cognition including automatic processes has a causal relationship with changes in more naturalistic observable behaviour (such as social interactions) and subjective feelings.

Despite these complexities, a consistent finding is that the behavioural and neurofunctional outcomes of exogenous oxytocin administration are highly dependent on the context and on variability in broadly defined 'individual differences factors' such as life experiences or personality traits. It has been suggested that rather than investigating whether oxytocin promotes social behaviour, the question should be when and for whom does oxytocin promote social behaviour (Bartz et al., 2011b). For example, contextual factors influencing oxytocin-induced response to social and emotional cues might be whether the faces stimuli are of familiar or unfamiliar individuals, or placed in a familiar or novel environment, and what are the motivational drivers within the situation (for example, cooperation or competition, task-relevance and ultimately even the expectations generated by the experimental setting). With regards to the influence of individual differences, it has been demonstrated that oxytocin's ability to modulate emotional processing could be dependent for example on participants' baseline levels of empathy (Bartz et al., 2010), social anxiety (Labuschagne et al., 2010, Clark-Elford et al., 2014) and early life stress (Grimm et al., 2014, Feeser et al., 2014). Future research should investigate whether variability in individual traits and life experiences corresponds to variations in endogenous oxytocin levels, which in turn may reflect variants in oxytocin receptor genes and their expression (see for example Puglia et al., 2015).

Finally, an additional factor that needs consideration in oxytocin research is sex-differences in the oxytonergic (and vasopressin) systems. Research in rodents suggests that oxytocin plays a sex-specific role in diverse social behaviors such as social avoidance, social recognition, partner preference, social play and social interest (for a review see Dumais and Veneema, 2015). While initial studies in humans were only conducted in male samples, more recent research has highlighted that gender differences may exist in the effects of oxytocin (Domes et al., 2007, 2010). Sex-specific differences in modulation of information processing may in turn impact on sex-dependent behavioural responses to oxytocin administration (Dumais and Veneema, 2015).

Progress in understanding the pharmacological and cognitive mechanism of action of oxytocin (and possibly most psychoactive substances) could then derive from new experimental designs that model 'if... then...' scenarios possibly with multiple interacting 'if' factors (Mischel and Shoda, 1995). Similar to individualised cognitive therapy formulations, one could test whether and how oxytocin may reduce anxiety in individuals with different attentional biases to social stimuli (such as preferential attention orientation to negative 
faces or attentional avoidance of all facial expressions) that map onto increasing levels of social anxiety, in the context of different task demands (for example, an evaluative vs. a supportive context). That pharmacological manipulation may produce different responses to emotional stimuli based on individual traits has also been shown for serotonergic antidepressants (Di Simplicio et al., 2014). In fact, while antidepressant reduction of negative biases in emotional processing has been more robustly replicated than any oxytocin-driven effect, utilising a more naturalistic 'if...then...' approach may help unravel some of the idiosyncrasies in the subjective response of clinical patients to antidepressant treatment that impact negatively on the drugs tolerability and therapy compliance; for example why and when do certain individuals respond to serotonergic drug administration with so-called 'emotional numbing' or 'paradoxical anxiety'.

This kind of individual approach has the potential for designing well combined pharmacological and behavioural / cognitive interventions: cognitive training or a behavioral intervention should produce the context whereby oxytocin exerts the desired prosocial effect given the individual's baseline characteristics. Although refining such interventions may appear quite laborious compared to the enthusiasm generated by some early translational applications (see clinical trials of oxytocin for schizophrenia, generalised anxiety and social anxiety, see Bakermans-Kranenburg and van IJzendoorn, 2013), this could represent an exciting avenue for experimental medicine designs. It could offer the chance of testing more targeted therapeutic interventions and avoiding an apparent lack of effects by averaging across different individual factors (see animal data on chronic oxytocin administration producing detrimental social effects, Bales et al., 2013).

In summary, research on oxytocin remains an exciting avenue for understanding social behaviour, brain function and for potential therapeutic application. Yet the burgeoning of research has revealed complex effects, which need to be explored in controlled studies particularly considering the role of different cognitive processes and individual differences. We believe that addressing this challenge will be and exciting opportunity for the next 30 years of psychopharmacology research to bring us closer to greater precision of psychiatric treatment and understanding.

\section{REFERENCES}

Andari E, Duhamel JR, Zalla T, et al. (2010). Promoting social behavior with oxytocin in highfunctioning autism spectrum disorders. Proc. Natl. Acad. Sci. 107, 4389-4394. doi:10.1073/pnas.0910249107

Bakermans-Kranenburg MJ, van IJzendoorn MH (2013). Sniffing around oxytocin: review and metaanalyses of trials in healthy and clinical groups with implications for pharmacotherapy. Translational Psychiatry (2013) 3, e258; doi:10.1038/tp.2013.34

Bales KL, Perkeybile AM, Conley OG, et al. (2013). Chronic Intranasal Oxytocin Causes Long-Term Impairments in Partner Preference Formation in Male Prairie Voles. Biol. Psychiatry 74, 180-188. doi:10.1016/j.biopsych.2012.08.025

Bartz JA, Zaki J, Bolger N, et al. (2010). Oxytocin Selectively Improves Empathic Accuracy. Psychol. Sci. $21,1426-1428$. doi:10.1177/0956797610383439

Bartz J, Simeon D, Hamilton H, et al. (2011a). Oxytocin can hinder trust and cooperation in borderline personality disorder. Soc. Cogn. Affect. Neurosci. 6, 556-563. doi:10.1093/scan/nsq085 
Bartz JA, Zaki J, Bolger N, et al. (2011b). Social effects of oxytocin in humans: context and person matter. Trends Cogn. Sci. 15, 301-309. doi:10.1016/j.tics.2011.05.002

Browning M, Reid C, Cowen PJ, et al. (2007). A single dose of citalopram increases fear recognition in healthy subjects. J. Psychopharmacol. 21, 684-690. doi:10.1177/0269881106074062

Chini B, Leonzino M, Braida D, et al. (2014). Learning About Oxytocin: Pharmacologic and Behavioral Issues. Biol. Psychiatry 76, 360-366. doi:10.1016/j.biopsych.2013.08.029

Clark-Elford R, Nathan PJ, Auyeung B, et al. (2015). Effects of Oxytocin on Attention to Emotional Faces in Healthy Volunteers and Highly Socially Anxious Males. Int. J. Neuropsychopharmacol. 31;18(2). pii: pyu012. doi: 10.1093/ijnp/py

Di Simplicio M, Norbury R, Reinecke A, et al. (2014). Paradoxical effects of short-term antidepressant treatment in fMRI emotional processing models in volunteers with high neuroticism. Psychol. Med. 44, 241-252.

Domes G, Heinrichs M, Glascher J, et al., (2007). Oxytocin attenuates amygdala responses to emotional faces regardless of valence Biol. Psychiatry. 62 (10), 1187-1190

Domes G, Lischke A, Berger C, et al. (2010). Effects of intranasal oxytocin on emotional face processing in women. Psychoneuroendocrinology 35, 83-93. doi:10.1016/j.psyneuen.2009.06.016

Domes G, Sibold M, Schulze L, et al. (2013). Intranasal oxytocin increases covert attention to positive social cues. Psychol. Med. 43, 1747-1753

Dumais KM, Veenema AH (2015). Vasopressin and oxytocin receptor systems in the brain: Sex differences and sex-specific regulation of social behavior, Frontiers in Neuroendocrinology, Available online 4 May 2015, ISSN 0091-3022, http://dx.doi.org/10.1016/j.yfrne.2015.04.003.

Ellenbogen MA, Linnen A-M, Cardoso C, et al. (2013). Intranasal oxytocin impedes the ability to ignore task-irrelevant facial expressions of sadness in students with depressive symptoms. Psychoneuroendocrinology 38, 387-398. doi:10.1016/j.psyneuen.2012.06.016

Ellenbogen MA, Linnen A-M, Cardoso C, et al. (2014). Intranasal oxytocin attenuates the human acoustic startle response independent of emotional modulation. Psychophysiology 51, 1169-1177. doi:10.1111/psyp.12263

Evans S, Shergill SS, Averbeck BB (2010). Oxytocin Decreases Aversion to Angry Faces in an Associative Learning Task. Neuropsychopharmacology 35, 2502-2509.

Feeser M, Fan Y, Weigand A, et al. (2014). The beneficial effect of oxytocin on avoidance-related facial emotion recognition depends on early life stress experience. Psychopharmacology (Berl). 231, 4735-4744. doi:10.1007/s00213-014-3631-1

Fischer-Shofty M, Shamay-Tsoory SG, Harari H, et al. (2010). The effect of intranasal administration of oxytocin on fear recognition. Neuropsychologia 48, 179-184. doi:http://dx.doi.org/10.1016/j.neuropsychologia.2009.09.003

Gamer M, Zurowski B, Büchel C (2010). Different amygdala subregions mediate valence-related and attentional effects of oxytocin in humans. Proc. Natl. Acad. Sci. 107 , 9400-9405. doi:10.1073/pnas.1000985107

Grillon C, Krimsky M, Charney DR, et al. (2013). Oxytocin increases anxiety to unpredictable threat. Mol Psychiatry 18, 958-960. 
Grimm S, Pestke K, Feeser M, et al. (2014). Early life stress modulates oxytocin effects on limbic system during acute psychosocial stress. Soc. Cogn. Affect. Neurosci. 9 , 1828-1835. doi:10.1093/scan/nsu020

Guastella AJ, Mitchell PB, Dadds MR (2008). Oxytocin Increases Gaze to the Eye Region of Human Faces. Biol. Psychiatry 63, 3-5. doi:10.1016/j.biopsych.2007.06.026

Guastella AJ, MacLeod C (2012). A critical review of the influence of oxytocin nasal spray on social cognition in humans: Evidence and future directions. Horm. Behav. 61, 410-418. doi:http://dx.doi.org/10.1016/i.yhbeh.2012.01.002

Harmer CJ, Bhagwagar Z, Perrett DI, et al. (2003). Acute SSRI Administration Affects the Processing of Social Cues in Healthy Volunteers. Neuropsychopharmacology 28, 148-152

Harmer CJ, Shelley NC, Cowen PJ, et al. (2004). Increased positive versus negative affective perception and memory in healthy volunteers following selective serotonin and norepinephrine reuptake inhibition. Am. J. Psychiatry 161, 1256-1263. doi:10.1176/appi.ajp.161.7.1256

Hurlemann R, Patin A, Onur OA, et al. (2010). Oxytocin Enhances Amygdala-Dependent, Socially Reinforced Learning and Emotional Empathy in Humans. J. Neurosci. 30 , 4999-5007. doi:10.1523/JNEUROSCI.5538-09.2010

Kosfeld M, Heinrichs M, Zak PJ, et al. (2005). Oxytocin increases trust in humans. Nature 435, 673677. doi:10.1038/nature03701

Labuschagne I, Phan KL, Wood A, et al. (2010). Oxytocin Attenuates Amygdala Reactivity to Fear in Generalized Social Anxiety Disorder. Neuropsychopharmacology 35, 2403-2413.

Leng G, Ludwig M (2016). Intranasal Oxytocin: Myths and Delusions. Biol. Psychiatry 79, 243-250. doi:10.1016/j.biopsych.2015.05.003

Lischke A, Berger C, Prehn K, et al. (2012a). Intranasal oxytocin enhances emotion recognition from dynamic facial expressions and leaves eye-gaze unaffected. Psychoneuroendocrinology 37, 475481. doi:10.1016/j.psyneuen.2011.07.015

Lischke A, Gamer M, Berger C, et al. (2012b). Oxytocin increases amygdala reactivity to threatening scenes in females. Psychoneuroendocrinology 37, 1431-1438. doi:10.1016/j.psyneuen.2012.01.011

Maroun M, Wagner S (2016). Oxytocin and Memory of Emotional Stimuli: Some Dance to Remember, Some Dance to Forget. Biol. Psychiatry 79, 203-212. doi:10.1016/j.biopsych.2015.07.016

Marsh AA, Yu HH, Pine DS, et al. (2010). Oxytocin improves specific recognition of positive facial expressions. Psychopharmacology (Berl). 209, 225-232. doi:10.1007/s00213-010-1780-4

Mischel W, Shoda Y (1995). A cognitive-affective system theory of personality: Reconceptualizing situations, dispositions, dynamics, and invariance in personality structure. Psychological Review, 102(2), 246-268. http://dx.doi.org/10.1037/0033-295X.102.2.246

Murphy SE, Downham C, Cowen PJ, et al. (2008). Direct effects of diazepam on emotional processing in healthy volunteers. Psychopharmacology (Berl). 199, 503-513. doi:10.1007/s00213008-1082-2

Neumann ID, Slattery DA (2016). Oxytocin in General Anxiety and Social Fear: A Translational Approach. Biol. Psychiatry 79, 213-221. doi:10.1016/j.biopsych.2015.06.004

Perry A, Bentin S, Shalev I, et al. (2010). Intranasal oxytocin modulates EEG mu/alpha and beta rhythms during perception of biological motion. Psychoneuroendocrinology 35, 1446-1453. doi:10.1016/j.psyneuen.2010.04.011 
Puglia MH, Lillard TS, Morris JP, et al. (2015). Epigenetic modification of the oxytocin receptor gene influences the perception of anger and fear in the human brain. Proc. Natl. Acad. Sci. $112,3308-$ 3313. doi:10.1073/pnas.1422096112

Shahrestani S, Kemp AH, Guastella AJ (2013). The impact of a single administration of intranasal oxytocin on the recognition of basic emotions in humans: a meta-analysis. Neuropsychopharmacology 38, 1929-36. doi:10.1038/npp.2013.86

Shamay-Tsoory SG, Fischer M, Dvash J, et al. (2009). Intranasal Administration of Oxytocin Increases Envy and Schadenfreude (Gloating). Biol. Psychiatry 66, 864-870. doi:10.1016/j.biopsych.2009.06.009

Shamay-Tsoory SG, Abu-Akel A (2016). The Social Salience Hypothesis of Oxytocin. Biol. Psychiatry 79, 194-202. doi:10.1016/j.biopsych.2015.07.020

Tollenaar MS, Chatzimanoli M, van der Wee NJA, et al. (2013). Enhanced orienting of attention in response to emotional gaze cues after oxytocin administration in healthy young men. Psychoneuroendocrinology 38, 1797-1802. doi:10.1016/j.psyneuen.2013.02.018

Walum H, Waldman ID, Young LJ (2016). Statistical and Methodological Considerations for the Interpretation of Intranasal Oxytocin Studies. Biol. Psychiatry 79, 251-257. doi:10.1016/j.biopsych.2015.06.016

Warren MB, Pringle A, Harmer CJ (2015). A neurocognitive model for understanding treatment action in depression. Philos. Trans. R. Soc. London B Biol. Sci. 370, (1677):20140213. doi: 10.1098/rstb.2014.0213 